Research Paper

\title{
Netrin-1 promotes cell neural invasion in gastric cancer via its receptor neogenin
}

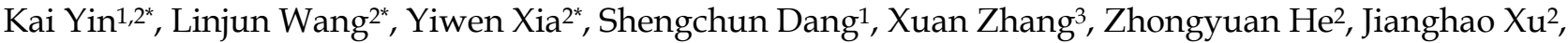 \\ Mengyuan Shang ${ }^{4}$, Zekuan $\mathrm{Xu}^{2 \bowtie}$ \\ 1. Department of General Surgery, Affiliated Hospital of Jiangsu University, Zhenjiang, Jiangsu, China \\ 2. Department of General Surgery, The First Affiliated Hospital of Nanjing Medical University, Nanjing, Jiangsu, China \\ 3. Department of General Surgery, The Affiliated Hospital of Xuzhou Medical University, Xuzhou, Jiangsu, China \\ 4. Department of Ultrasound Diagnosis, Affiliated Hospital of Jiangsu University, Zhenjiang, Jiangsu, China \\ "These authors contributed equally to this work. \\ $\triangle$ Corresponding authors: Zekuan Xu, The First Affiliated Hospital of Nanjing Medical University, 300 Guangzhou Road, Nanjing, Jiangsu, China, Email: \\ xuzekuan@njmu.edu.cn; Mengyuan Shang, Affiliated Hospital of Jiangsu University, 438 Jiefang Road, Zhenjiang, Jiangsu, China, Email: \\ shangmy1985@sina.com \\ (c) Ivyspring International Publisher. This is an open access article distributed under the terms of the Creative Commons Attribution (CC BY-NC) license \\ (https://creativecommons.org/licenses/by-nc/4.0/). See http://ivyspring.com/terms for full terms and conditions.
}

Received: 2018.09.27; Accepted: 2019.04.30; Published: 2019.06.02

\begin{abstract}
Neural invasion $(\mathrm{NI})$ is one of the important routes for local spread of gastric cancer (GC) correlated with poor prognosis. However, the exact cellular characteristics and molecular mechanisms of $\mathrm{NI}$ in $\mathrm{GC}$ are still unclear. Netrin-1(NTNI) as an axon guidance molecule was firstly found during neural system development. Importantly, NTNI has an essential role in the progression of malignant tumor and specifically mediates the induction of invasion. In this study, we found NTN1 expression was significantly increased in 97 tumor tissues from GC patients and positively correlated with $\mathrm{NI}(\mathrm{p}<0.05)$. In addition, we detected NTNI knockdown significantly suppressed GC cells migration and invasion. Moreover, our results showed that reciprocity was observed between GC cells and neurites colonies in dorsal root ganglia (DRG)-GC cells co-culture vitro model. GC cells with NTNI silencing could suppress their abilities to navigate along surrounding neuritis and this effect was depended on its receptor neogenin. In vivo, NTN1 inhibition also decreased GC cells sciatic nerve invasion. Taken together, our findings argue that NTN1 and its receptor neogenin might act synergistically in promoting GC cells neural invasion. Inhibiting the activity of NTN1 could be a potential strategy targeting $\mathrm{NI}$ in GC therapy.
\end{abstract}

Key words: Netrin-1; Gastric cancer; Neural invasion; Neogenin

\section{Introduction}

Gastric cancer (GC) is now the third leading cause of cancer related death in male and fifth in female worldwide [1]. Neural invasion (NI) is thought to be one of the factors responsible for the high rate of tumor recurrence after surgery and the pain generation associated with GC. NI in GC has been shown an important but less studied mechanism of metastasis.

NI is a process which was described as the spread of cancer cells in the perineural spaces of the nerves. NI is a well-known route of cancer spread in malignant diseases, such as the head/neck cancer [2], prostate cancer [3, 4], colorectal cancer [5] and pancreatic cancer [6-9]. It was reported to be a crucial route for local spread of cancers and related to cancer recurrence, cancerous pain and poor prognosis [10]. Dissemination of cancer cells into the nerves has been shown in up to $50-60 \%$ of GC patients [11]. NI represents a key pathologic feature of GC, it is an established prognostic factor for GC. Thus, we need a clear clinical imperative to improve the understanding of the molecular mechanisms underlying NI in GC.

Although lots of studies on NI have been carried out in many types of cancer, the molecular mechanisms about the interaction between GC cells and neural cells remain poorly understood. Netrin-1(NTN1) was a laminin-like protein, originally 
identified as an axon guidance molecule during neural system development [12]. NTN1 activity was mediated by several receptors, including uncoordinated5A-D (UNC5A-D), deleted in colorectal cancer (DCC), its orthologue neogenin, and down syndrome cell adhesion molecule (DSCAM). In tumors, NTN1 acted an oncogene which was upredulated in neuroblastoma [13], pancreatic cancer $[8,14]$, breast cancer [15], prostate cancer [16] and non-small cell lung carcinoma [17]. Additionally, NTN1 has been identified as a novel stimulator of cancer cell invasion in hepatic carcinoma and colorectal cancer $[18,19]$. Our previous study has proved that NTN1 promoted GC cells migration and invasion via its receptor neogenin [20]. The focus of this study was to provide a comprehensive characterization of NI in GC with emphasis on its underlying molecular mechanisms, and whether NTN1 and neogenin were involved in this process.

In this study, we found that NTN1 was expressed in GC tissues and this expression was significantly associated with NI. To detect the molecular mechanisms between GC cells and neural cells, we established co-culture model between GC cells and dorsal root ganglia (DRG) in the three-dimensional(3D)-neural-migration assay in vitro. Here, we demonstrated that NTN1 and its receptor neogenin played an important role in mediating GC cells interaction with neural cells.

\section{Materials and Methods}

\section{Tissue samples and cell culture}

In total, 97 GC samples and matched non-tumor tissues were collected at the time of surgical resection between 2014 and 2016 at the first affiliated hospital of Nanjing medical university and affiliated hospital of Jiangsu university. All GC patients were diagnosed with primary gastric cancer and none of the patients had a history of radiotherapy or chemotherapy prior to surgery. The patients with distant metastasis at diagnosis were excluded from the study. The eligibility criteria consisted of histologically confirmed R0 gastric resection, which was defined as no microscopic residual tumor. GC with NI was examined by 3 different tissue specimens from the main tumor. Histopathologic analysis was conducted by 2 independent observers blinded to patient diagnosis. Written informed consent was obtained from all the patients. The study was approved by the Ethics Committee of affiliated hospital of Jiangsu University.

Human GC cell lines (BGC823, MGC803, MKN28, SGC7901 and MKN45) and gastric mucosa epithelial cell line (GES1) were purchased from the
Cell Bank of Chinese Academy of Medical Science (Shanghai, China). These cells were cultured in 1640 medium containing $10 \%$ fetal bovine serum (Invitrogen Life Technology, CA, USA), penicilin $(100 \mathrm{U} / \mathrm{ml})$, and streptomycin $(100 \mathrm{mg} / \mathrm{ml})$ at $37^{\circ} \mathrm{C}$ with $5 \% \mathrm{CO} 2$.

\section{Quantitative real-time PCR}

Total RNA was extracted from GC cells and GC tissues with TRIzol reagent (Invitrogen Life Technology, Waltham, MA, USA) and cDNA was synthesized using PrimeScript RT Reagent (Takara, Dalian, China) according to the manufacturer's instructions. The quantitative real-time PCR were performed as previously described [21]. The FastStart Universal SYBR Green Master (ROX) (Roche, Mannheim, Germany) was used in real-time PCR in an Applied Biosystems 7500 sequence detection system. The qRT-PCR primers were as follows: NTN1, forward, 5'-AAGCAGGGCACAAGTCGTAT-3' and reverse, 5'-TGCTCTTGTCTGCCACGATG-3'; UNC5A, forward, 5'-CCGGCTGATGATCCCTAA TA-3' and reverse, 5'-CTTGTGCAGCGTGAGGTA GA-3'; UNC5B, forward, 5'-GAGGTGGAATGGCT CAAGAA-3' and reverse, 5'-ATGAGGTTGTGGT CGATGGT-3'; UNC5C, forward, 5'-AGCAAGGCAG ACTGATCCAT-3' and reverse, 5'-TCAGCAAGCT GACTCCTGAA-3'; UNC5D, forward, 5'-AGTGGGT CCATCAGAACGAG-3' and reverse, 5'-CATGGA AGTCCTCCACCTGT-3'; DCC, forward, 5'-GCCACA AACCAACAGAGGAT-3' and reverse, 5'-GCTGCTT CATGAGTCCTTCC-3'; Neogenin, forward, 5'-ATGG TGACCAAAGGTCGAAG-3' and reverse, 5'-AGTCA CATCCTTGGGTGGAG-3'; DSCAM, forward, 5'-TCC ACCTCAGGAAGTTCACC-3' and reverse, 5'-CCACG GATAATCCCATTTTG-3'; $\beta$-actin, forward, 5'-TTAG TTGCGTTACACCTTTC-3' and reverse, 5'-ACCTT CACCGTTCCAGTTT-3'. Levels of gene expression were determined by $\triangle \triangle C T$ method, with the results being expressed as mRNA expression levels normalized to the levels of $\beta$-actin.

\section{Immunohistochemical analysis}

All GC tissues and adjacent normal tissues were fixed in $4 \%$ formalin and then embedded in paraffin. The $4 \mu \mathrm{m}$ of tissue slides were incubated with diluted primary antibody against Netrin-1 (NO.ab122903, Abcam, Cambridge, UK) and neogenin (NO.HPA027806, Sigma-Aldrich, USA). Reaction products were performed by 3, 3-diaminobenzidine (DAB) solution and the nuclei were counterstained with haematoxylin. Expression levels of Netrin-1 and neogenin were evaluated according to the staining intensity ( 0 for absent, 1 for weak, 2 for moderate and 3 for strong staining). The following scores were used 
to describe the overall proportion of Netrin-1 and neogenin positive cells: 0 (negative), $1(<30 \%), 2$ $(31-60 \%), 3(>60 \%)$. The two scores were multiplied, and the scores $\geq 4$ were defined as high expression, and scores $<4$ were defined as low expression.

\section{RNA interference, plasmids and lentivirus transfection}

The shRNA targeting NTN1 (shNTN1-1:5'CATGGAGCTCTACAAGCTT-3' and shNTN1-2: 5'-GCCUGCAAAGCCUGUGAUUTT-3') and the scramble shRNA (5'-GTTCTCCGAACGTGTCA CGT-3') were packaged in lentivirus vectors (GenePharma, Shanghai, China). The transfected cells were selected with $5 \mu \mathrm{g} / \mathrm{ml}$ puromycin (SigmaAldrich, St Louis, MO, USA) for 7 days to build stable cell lines. The siRNA duplexes targeting neogenin (siNeo: 5'-GCUGUUUGGUGUAGGUAAA-3') and a control siRNA (siCTL: 5'-TTCTCCGAACGTGTCA CGTTT-3') were also purchased from GenePharma and transfected into GC cells using Lipofectamine 3000 reagent (Invitrogen Life Technology, CA, USA). All transfections were performed according to the manufacturer's instructions.

\section{Wound healing assay}

Briefly, $6 \times 10^{5}$ GC cells were seeded in 6-well Petri dishes for $48 \mathrm{~h}$ and allowed to reach approximately $90 \%$ confluence. A scratch was made in each well using sterile $100-\mu l$ pipet tips. Subsequently, then the cells were washed by PBS and incubated in growth medium for $24 \mathrm{~h}$ at $37^{\circ} \mathrm{C}$ with $5 \% \% \mathrm{CO} 2$. After $24 \mathrm{~h}$, the scratches were documented and the area between both wound sides was calculated. The migration area was analyzed with the ImageJ program. The experiments were performed in triplicate.

\section{Transwell migration and invasion assays}

Cell invasion and migration assays were analyzed using the Transwell (Corning Costar, New York, NY, USA) assay, with or without coated Matrigel (BD Biosciences, Franklin Lakes, NJ, USA). Briefly, a total of $3 \times 10^{4}$ cells (MKN28 and MGC803) in $200 \mu \mathrm{l}$ serum-free 1640 medium were plated in the upper chambers, the lower chamber was filled with $600 \mu 11640$ medium containing 10\% FBS. After 24 hours of incubation, cells invading into the bottom side of the inserts were fixed and stained with crystal violet dye. The migrated or invaded cells in the lower chambers were observed under an electron microscope.

\section{Western blotting assay}

Western blotting was performed as previously described by us [22]. The following primary antibodies were used: Netrin-1 (NO.ab122903, Abcam, Cambridge, UK; 1:500 dilution), neogenin (NO.HPA027806, Sigma-Aldrich, USA), GAPDH (NO.51332, Cell Signaling Technology, Danvers, MA, USA; 1:1000 dilution). GAPDH was used as an internal control. The total proteins were analyzed on the $10 \%$ SDS polyacrylamide gels by SDS-PAGE and transferred onto PVDF membranes (Millipore, Bedford, MA, USA). After being blocked with 5\% non-fat powdered milk for $2 \mathrm{~h}$, the membranes were incubated with specific primary antibodies at $4{ }^{\circ} \mathrm{C}$ overnight and washed with TBST 15 min 3 times, then the blotted membranes were incubated with secondary antibodies (GeneTex, San Antonio, TX, USA) for $2 \mathrm{~h}$ at room temperature. Targeting protein expression levels were visualized using an enhanced chemiluminescence kit (Millipore, Temecula, CA, USA) and detected with an enhanced chemiluminescence detection system (FluorChem E, ProteinSimple, San Jose, CA, USA).

\section{DRG-tumor cells co-culture assay}

DRG-tumor cells co-culture assay was performed using a modified method based on a previously described protocol [23]. Briefly, $2 \times 10^{5}$ MGC803 cells were suspended in $25 \mu 1$ of growth-factor-reduced Matrigel (Sigma-Aldrich, St Louis, MO, USA) and placed at the centre of each well of 12-well Petri dishes. DRGs were isolated from the lumbar spinal region of 8-day-old Sprague-Dawley rats and seeded at $1 \mathrm{~mm}$ distance to the cell suspension. To exclude the possibility of non-specifically guided migration of GC cells, an additional $25 \mu$ l of blank Matrigel was positioned on the opposite side. The Petri dishes were incubated for 30 minutes at $37^{\circ} \mathrm{C}$ in a humidified $5 \% \mathrm{CO} 2$-saturated atmosphere to allow Matrigel polymerization. After solidification, neurobasal medium (Gibco, Carlsbad, USA) supplemented with $10 \%$ FBS, $100 \mathrm{U} / \mathrm{ml}$ penicillin, $100 \mu \mathrm{g} / \mathrm{ml}$ streptomycin, $0.5 \mathrm{mM}$ L-glutamine and 2\% B-27 (Invitrogen, Carlsbad, CA, USA) were added to the wells and renewed every 2 days. Travel distances, velocity, and directness were calculated using ImageJ software.

\section{Sciatic nerve invasion assay in vivo}

Four-week-old male nude mice (BALB/c nude mice) were purchased from the Department of Laboratory Animal Centre of Nanjing Medical University. $2 \times 10^{5}$ MGC803 cells were injected into the periphery of the sciatic nerve by using a dissecting microscope and 33 gauge needle in 5 $\mu$ PBS. MGC803 cells with NTN1 knockdown were injected into the right pygal and the control cells were injected into the left pygal. Mice were observed for 6 weeks for gross 
behavior, limb function, and sciatic nerve function, as determined by paw spread distance. The xenograft diameters were measured using a slide caliper every other day until day42. The xenograft tumor volume was calculated using the following formula: $v=0.5 \mathrm{ab}^{2}$ $(\mathrm{a}=$ the long diameter of the tumor, $\mathrm{b}=$ the short diameter of the tumor, and v= volume) [24]. Six weeks later, mice were subjected to magnetic resonance imaging, performed on a Bruker USR 7.0-T40-cm bore scanner (Bruker Biospin MRI, Inc, Billerica, MA) using a custom-designed active decoupled radiofrequency surface coil (Stark MRI Contrast Research, Erlangen, Germany) to improve signal to noise ratio and spatial resolution. Care of experimental animals was in accordance with Nanjing Medical University Institutional Animal Care and Use Committee.

\section{Statistical analysis}

The statistical analyses were performed using SPSS version 22.0. The data are presented as the mean \pm standard error of the mean (SEM) unless indicated otherwise. The statistical significance of differences between two groups was evaluated by the paired Student $\mathrm{t}$-test. Data from more than two groups were analyzed using two-way ANOVA. Correlation between NTN1 expression level and clinicopathological factors was performed by Pearson $X^{2}$ test. $\mathrm{P}$ values less than 0.05 were considered to be statistically.

\section{Results}

\section{NTN1 was overexpressed in GC tissues and its expression correlated with NI and lymph node metastasis}

NI was detected in 58 of 97 GC patients $(59.8 \%)$ and these patients showed varying degrees of NI. To explore whether NTN1 was upregulated in GC tissues, we collected 97 pairs of tumor and adjacent normal tissues to detect the expression level of NTN1 by using qRT-PCR. Our dates showed that NTN1 expression level was higher in GC tumor tissues compared with the matched adjacent normal tissues (Fig. 1A). We next analyzed the correlation between the expression level of NTN1 and clinicopathological characteristics of GC patients. Our findings showed that increased NTN1 in GC tissues was significantly correlated with NI and lymph node metastasis (Table 1). In addition, IHC staining also confirmed that NTN1 protein was almost absent in normal gastric tissue but aberrantly elevated in GC tissues. Importantly, we found NTN1 staining was further increased in GC tissues with NI (Fig. 1B-C).

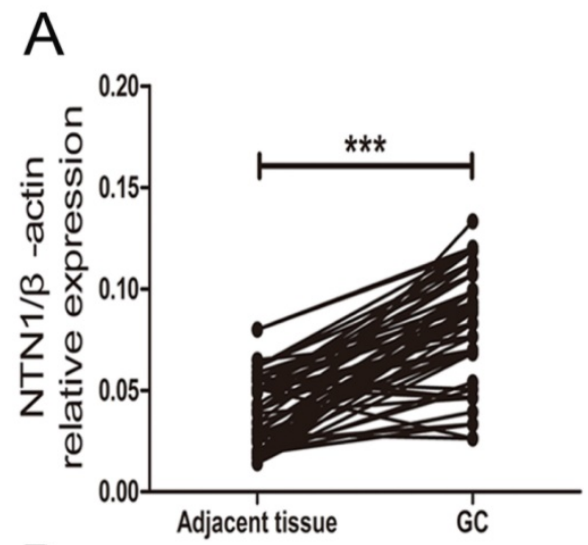

$\mathrm{B}$

Adjacent tissue
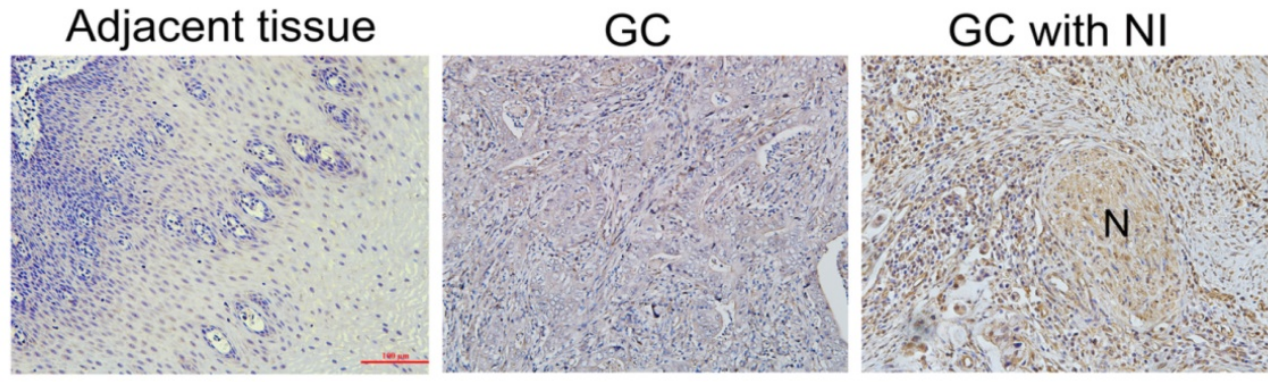

Fig.1. The expression level of NTN1 was increased in GC specimens. A. NTN1 mRNA expression level in 97 paired GC tissues and adjacent normal tissues were investigated by qRT-PCR. B. Representative results of upregulation of NTN1 protein in GC specimens with or without NI by immunohistochemistry. N indicated nerve. Original magnification, 200x; Scale bar $=100 \mu \mathrm{m}$. C. Quantification of the percentage of cells demonstrating the presence of immunoreactivity for NTN1 (positive staining). *p $<0.05$, $* * \mathrm{p}$ $<0.01, * * * \mathrm{p}<0.001$ 


\section{NTN1 knockdown suppressed GC cells migration abilities in vitro}

We examined the expression of NTN1 in normal gastric mucosa epithelial cell (GES1) and GC cells lines, including BGC823, MGC803, MKN28, SGC7901 and MKN45 by qRT-PCR. We found GC cell lines (MGC803 and MKN28) had the highest levels of NTN1, BGC823 and SGC7901 cell lines expressed NTN1 at moderate levels, while MKN45 and GES1 had no expression level of NTN1 (Fig. 2A). In ordered to study the role of NTN1 in GC cells motility, we inhibited NTN1 expression in MGC803 and MKN28 cell lines by using two different shRNA. As shown in Fig. 2B, each GC cell line transfected with NTN1 lentivirus showed efficient silencing of NTN1 expression, as determined by western blotting and qRT-PCR. The wound healing was carried out to explore the effect of NTN1 on the migration of MGC803 and MKN28 cells. Our results indicated that the gap sizes of MGC803 (Fig. 2C-D) and MKN28 (Fig. 2E-F) cells with NTN1 inhibition were significantly larger than negative control cells. These results suggested that migration abilities of MGC803 and MKN28 cells were decreased abundantly after NTN1 silencing.

\section{NTN1 knockdown suppressed GC cells invasion abilities in vitro}

Then we conducted Transwell assay to further illustrate the impact of NTN1 on migration and invasion abilities of GC cells. We discovered that NTN1 knockdown markedly reduced the number of migrated MGC803 and MKN28 cells (Fig. 3A-B). Furthermore, the number of invasive MGC803 and MKN28 cells with NTN1 inhibition obviously decreased compared with negative control cells (Fig. 3C-D). In a word, our date suggested that NTN1 knockdown inhibited GC cells migration and invasion abilities in vitro.

\section{NTN1 knockdown suppressed the migratory ability along the nerve of GC cells in a DRG-GC cells co-culture assay}

To identify GC cells that have a propensity to invade nerves, a modified co-culture assay of DRG and MGC803 cells was established. After the two cells were co-cultured for approximately 5 days, MGC 803 cells dissociated from colonies and formed clusters which migrated in a unidirectional fashion along the nerve toward the ganglion. On the 7 th day after starting co-cultured, we evaluated the accumulated travel distance and migrating velocities of the cancer cells (Fig. 4A-B). We showed MGC803 control cells reached a higher migrating velocity than MGC803 cells with NTN1 inhibition (Fig. 4C). Moreover, MGC803 control cells travelled a greater distance compared with MGC803 shNTN1 cells (Fig. 4D). In addition, we analyzed the neurites that grew out from the DRGs. Our results demonstrated that the average area covered by the neurites that co-cultured with MGC803 cells with NTN1 inhibition was smaller compared with that co-cultured with control cells (Fig. 4E). These results indicated that NTN1 knockdown suppressed the migratory potential of GC cells along the nerve.

Table 1. Correlation of relative NTN1 expression with clinicopathological characteristics of gastric cancer patients

\begin{tabular}{|c|c|c|c|c|}
\hline \multirow[t]{2}{*}{ Characteristics } & \multirow[t]{2}{*}{ Number (\%) } & \multicolumn{2}{|c|}{ NTN1 expression } & \multirow[t]{2}{*}{$p$-value } \\
\hline & & \multicolumn{2}{|c|}{ Low group High group } & \\
\hline \multicolumn{5}{|l|}{ Age(years) } \\
\hline$>60$ & $51(52.6)$ & 30 & 21 & 0.990 \\
\hline$\leq 60$ & $46(47.4)$ & 27 & 19 & \\
\hline \multicolumn{5}{|l|}{ Gender } \\
\hline Male & $60(61.9)$ & 25 & 35 & 0.235 \\
\hline Female & $37(38.1)$ & 20 & 17 & \\
\hline \multicolumn{5}{|l|}{ Size $(\mathrm{cm})$} \\
\hline$<3 \mathrm{~cm}$ & $42(43.3)$ & 23 & 19 & 0.842 \\
\hline$\geq 3 \mathrm{~cm}$ & $55(56.7)$ & 29 & 26 & \\
\hline \multicolumn{5}{|l|}{$\mathrm{T}$ grade } \\
\hline $\mathrm{T} 1+\mathrm{T} 2$ & $35(36.1)$ & 17 & 18 & 0.207 \\
\hline $\mathrm{T} 3+\mathrm{T} 4$ & $62(63.9)$ & 22 & 40 & \\
\hline \multicolumn{5}{|c|}{ Lymph node metastasis } \\
\hline No & $33(34.0)$ & 21 & 12 & $0.045^{*}$ \\
\hline N1-N3 & $64(66.0)$ & 27 & 37 & \\
\hline \multicolumn{5}{|l|}{ Stage } \\
\hline $\mathrm{I} / \mathrm{II}$ & $37(38.1)$ & 19 & 18 & 0.274 \\
\hline III/IV & $60(61.9)$ & 24 & 36 & \\
\hline \multicolumn{5}{|l|}{ Histological type } \\
\hline Intestinal & $42(43.3)$ & 19 & 23 & 0.983 \\
\hline Diffuse & $55(56.7)$ & 25 & 30 & \\
\hline \multicolumn{5}{|c|}{ Tumor differentiation } \\
\hline Well-Moderately & $45(46.4)$ & 18 & 27 & 0.173 \\
\hline Poorly-signet & $52(53.6)$ & 28 & 24 & \\
\hline \multicolumn{5}{|l|}{ Neural invasion } \\
\hline Absent & $39(40.2)$ & 21 & 18 & $0.001^{\star *}$ \\
\hline Present & $58(59.8)$ & 13 & 45 & \\
\hline
\end{tabular}

${ }^{*} \mathrm{P}<0.05 ; *{ }^{*} \mathrm{P}<0.01$.

\section{NTN1 knockdown suppressed GC cells NI ability was depend on neogenin}

Since inhibition of NTN1 results in a decrease in the migration of GC cells towards the neurites, we performed a reciprocal experiment to determine whether its receptor was responsible for this process. Reportedly, neogenin was detected to upregulated in GC tissues and increased invasion abilities of GC cells [25]. We also found neogenin expression level in GC tissues was higher than that in adjacent tissues by IHC staining (Fig. 5A-B). Then we investigated the expression level of NTN1 receptors including UNC5A-D, neogenin, DCC and DSCAM in MGC803 and MKN28 cells by qRT- PCR, and found neogenin was the highest expressed in these cells (Fig. 5C).

Therefore, we inhibited neogenin (named siNeo) in MGC803 and MKN28 cells and western blotting 
showed that the siRNA efficiently reduced the expression level of neogenin (Fig. 5D). Our previous studies have demonstrated that knockdown neogenin can reduce GC cells migration and invasion abilities [20]. In the present study, we found silencing neogenin also reduced the invasive abilities of MGC803 cells towards the DRG (Fig. 5E). Moreover, our results showed that MGC803 negative control cells reached a higher migrating velocity than
MGC803 cells with neogenin inhibition (Fig. 5F). Similarly, we found MGC803 negative control cells travelled a greater distance compared with MGC803 cells with neogenin inhibition and the area covered by neurites in neogenin inhibition group was smaller than that in control group (Fig. 5G-H). Thus, we hypothesized that NTN1/neogenin signaling pathway might play an important role in GC cells neural invasion.
A

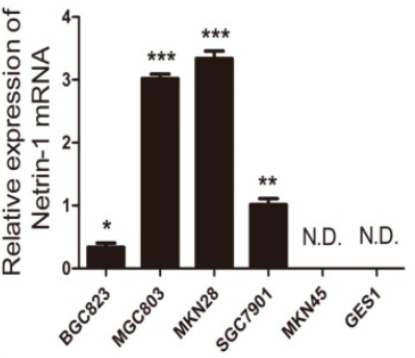

$\mathrm{c}$

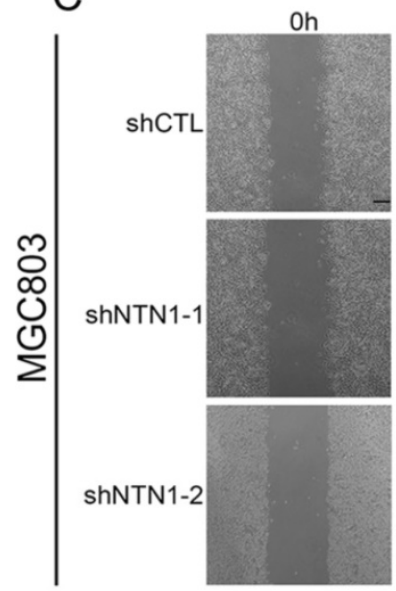

E
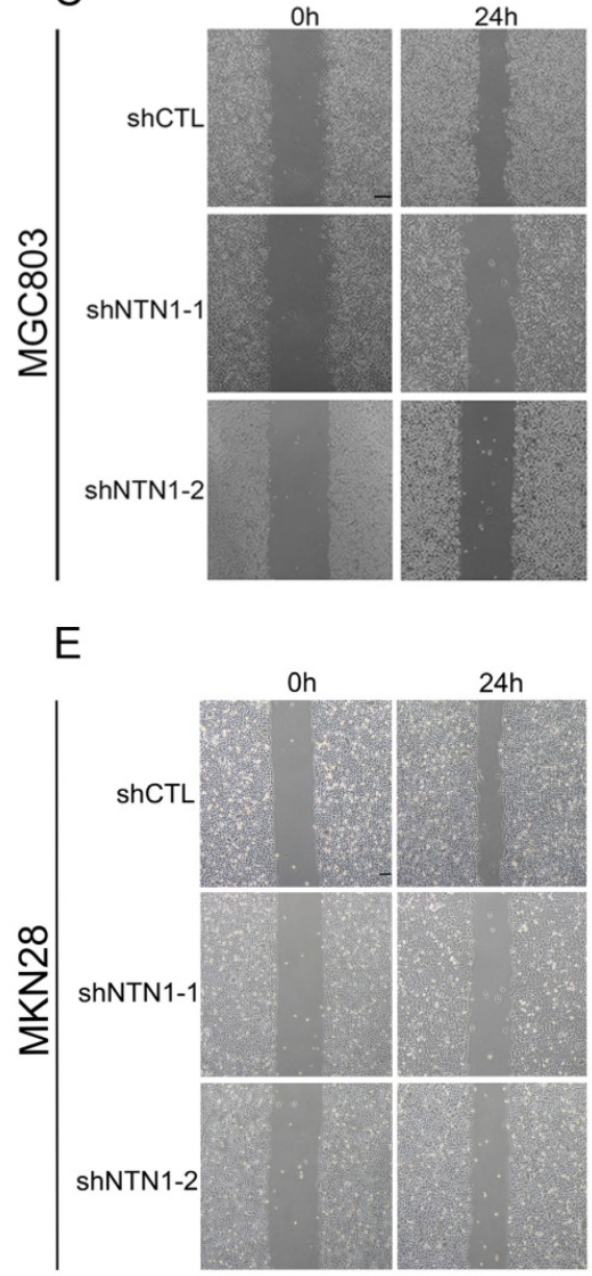

B

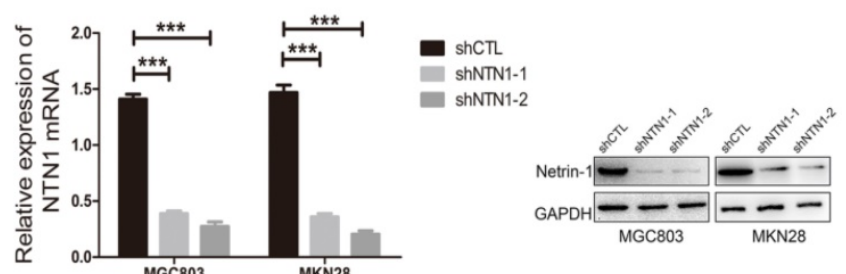

D

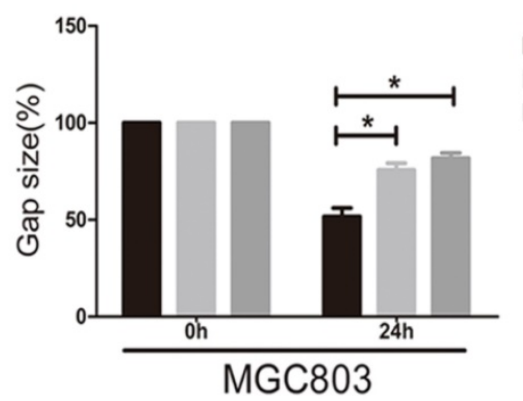

F

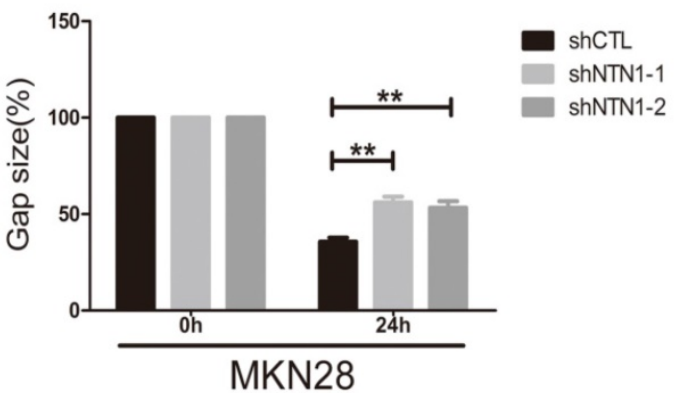

Fig. 2. NTN1 knockdown inhibited GC cells migration abilities in vitro. A. The expression of NTN1 was measured using qRT-PCR in five GC cell lines (BGC823, MGC803, MKN28, SGC7901 and MKN45) and compared with that measured in the gastric mucosa epithelial cell line GES1. B. NTN1 was efficiently decreased by NTN1 shRNA in MGC803 and MKN28 cells. NTN1 expression level was examined by western blotting and qRT-PCR after transfection for 48 hours. C-D. NTN1 knockdown slowed the wound healing in MGC803 cells. The gap size was measured and plotted as the percentage of the original time point ( 0 hour). Representative images of wound healing assays were shown. Original magnification, 40x; Scale bar $=100 \mu \mathrm{m}$. E-F. NTN1 knockdown slowed the wound healing in MKN28 cells. The gap size was measured and plotted as the percentage of the original time point ( 0 hour). Representative images of wound healing assays were shown. Original magnification, $40 \times ;$ Scale bar $=100 \mu \mathrm{m} .{ }^{*} \mathrm{p}<0.05, * * \mathrm{p}<0.01$, $* * * \mathrm{p}<0.001$. 

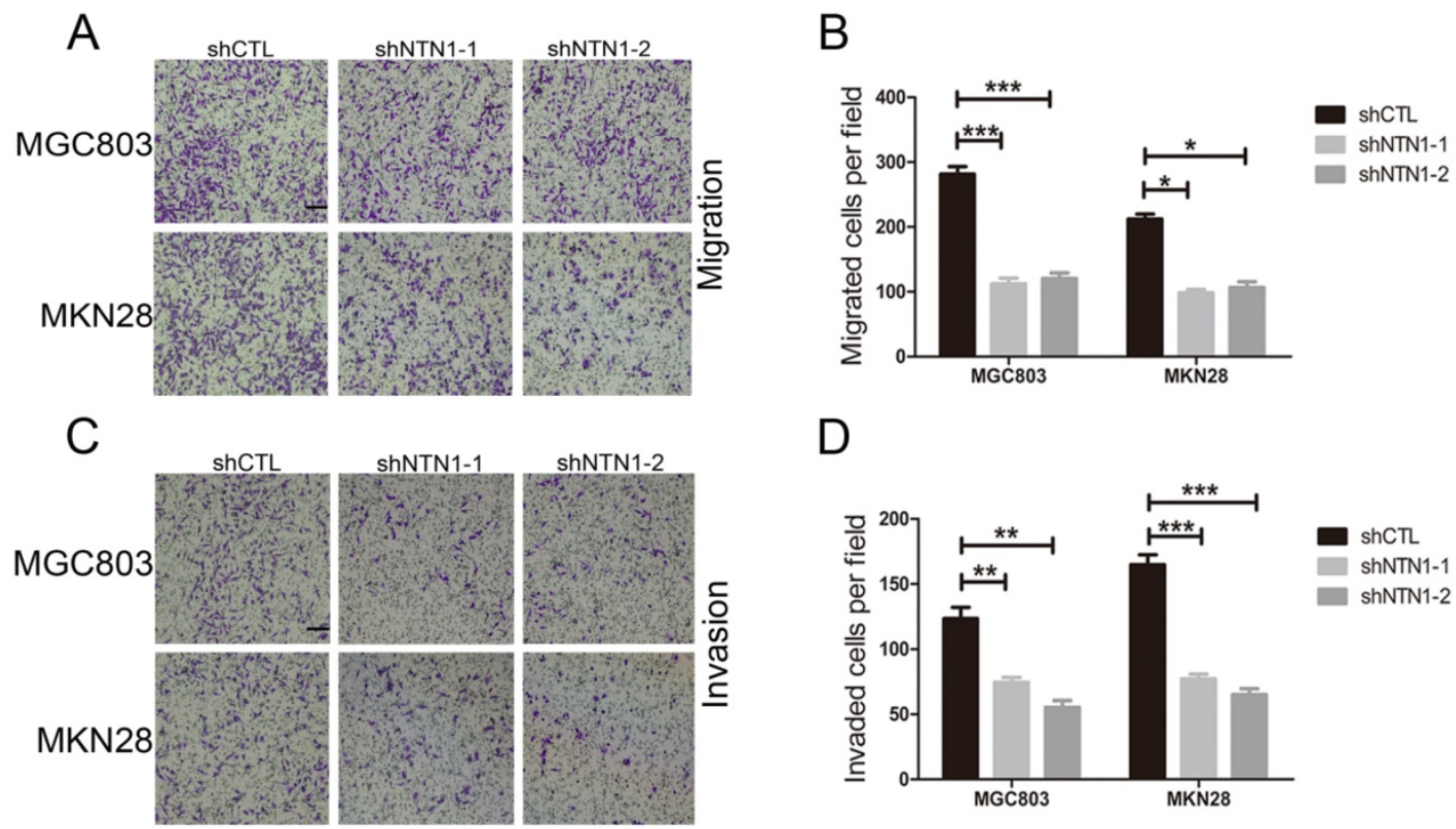

Fig. 3. NTN1 knockdown inhibited GC cells invasion abilities in vitro. A-B. NTN1 knockdown inhibited MGC803 and MKN28 cells migration abilities in Transwell assay. Representative images are shown. Original magnification, 100x; Scale bar $=100 \mu \mathrm{m}$. The number of migrated cells was quantified. C-D. The invasive capabilities of MGC803 and MKN28 cells were investigated by Matrigel-coated Transwell assay. Representative images are shown. Original magnification, 100x; Scale bar = 100 $\mu m$. The number of invasive cells was quantified. ${ }^{*} \mathrm{p}<0.05, * * \mathrm{p}<0.01,{ }^{*} * \mathrm{p} p<0.001$.

\section{Inhibition of NTN1 decreased NI of GC cells in vivo}

We investigated NTN1 ability to regulate NI by injecting MGC803 cells (control and shNTN1) into the periphery of sciatic nerve (left and right) in nude mice respectively. Our results showed that mice began to develop left hind limb paralysis 4 weeks after tumor implantation (Fig. 6A). In contrast, NTN1 knockdown group had normal right hind limb function until week 6. Histopathologic evaluation (HE staining) revealed that GC cells extensive invaded proximal nerve from the primary tumor (Fig. 6B-C). Nerve function was determined by paw spread, where at week 0 both NTN1 inhibition and negative control groups had a paw spread of $9.85 \pm 0.25$ and 0.26 , respectively. Six weeks later, the control group paw spread decreased to $3.40 \pm 0.40$, but in NTN1 inhibitor group only decreased to $7.40 \pm 0.42$ (Fig. 6D). In addition, tumor volume and proximal nerve diameter in different groups were measured using small animal MRI (Fig. 6E-F). Tumor growth curves showed that the tumor volumes in the sciatic nerve in NTN1 knockdown group were significantly smaller compared with the control group (Fig. 6G). Moreover, our results showed that proximal nerve diameter in the control group was larger compared with that in NTN1 knockdown group (Fig. 6H). In a word, GC cells with NTN1 knockdown inhibited their invasion abilities through the nerve and then protected nerve function. Thus, our dates indicated that GC cells neural invasion ability was impaired after NTN1 silencing.

\section{Discussion}

NI was the process through which cancer cells invade and extend along nerves. NI was reported to be a crucial pattern of invasion and a characteristic pathological feature of GC, which related to poor progression and prognosis in GC [26]. In this study, we observed the expression level of NTN1 was higher in human GC tissues and this expression was association with NI and lymph node metastasis. Our in vitro and in vivo data, furthermore, demonstrated a novel function to NTN1, as they implicated NTN1 in the control of GC cells invasion along outgrowing neurites. To best of our knowledge, this is the first report that NTN1 involved in NI of GC.

NTN1 has been considered as an oncogene in many types of malignant tumor [27]. The promoting role of NTN1 on cancer progression was mostly focused on regulating cells proliferation, angiogenesis, apoptosis, migration and invasion $[8$, 28-32]. In this study, we also showed NTN1 could promote GC cells migration and invasion abilities by wound healing assay and Transwell assay, while down-regulation of NTN1 inhibited GC cells migration and invasion abilities. In addition to its roles in cancer progression, NTN1 was also involved in nerve development, nerve injury repair and neuropathic pain [33]. NTN1 expression was elevated in Schwann cells and promoted cells migration through P38/MAPK and PI3K/AKT signaling pathway [34]. Interestingly, we identified that the 
A
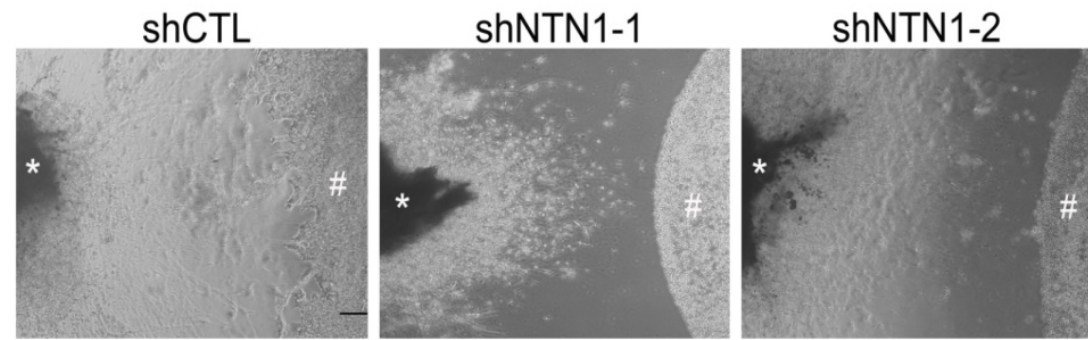

B

Day3

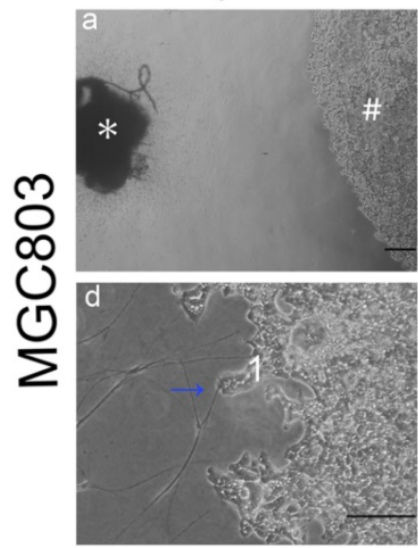

Day7

C

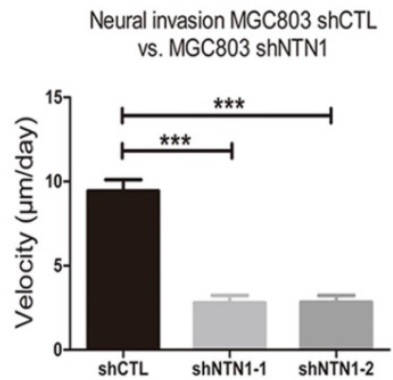

Day5
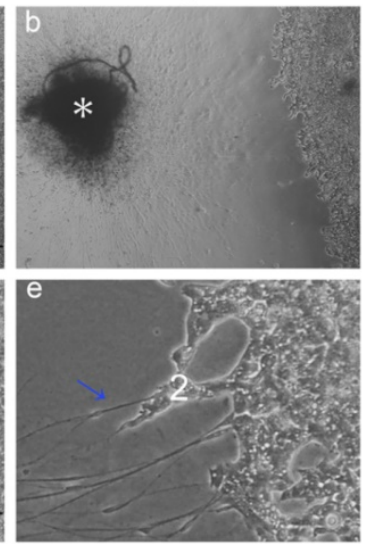

Day7

D

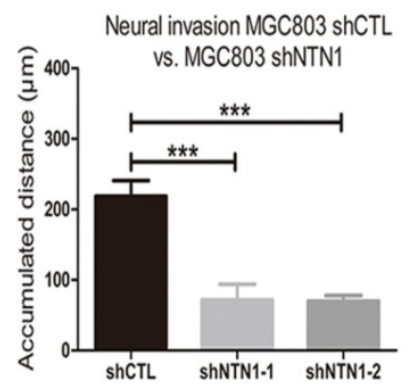

Day7

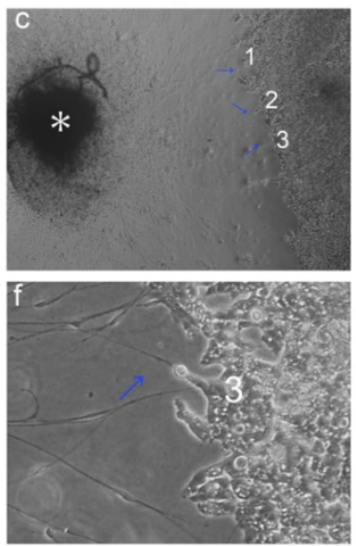

Day7

$E$

Fig. 4. NTN1 knockdown suppressed GC cells neural invasion in DRG-tumor cell co-culture assay. A. Representative images of co-cultured with MGC803 control cells or MGC803 shNTN1 cells in the DRG-tumor cell co-culture assay. (\#) indicates GC cells, $(*)$ indicates DRG. Original magnification, $40 \times$; Scale bar $=100 \mu m$. B. Representative photomicrographs showing the entire process of the DRG-tumor cell interaction. (a-b) Three-dimensional co-culture of GC cells (\#) and DRG (*) were assembled in Matrigel. (c) Outgrowth and extension (blue arrowheads) of neurites from the DRG toward the tumor cell colony around day 7. Original magnification, 40×; Scale bar $=100 \mu \mathrm{m}$. The lower panel showed that GC cells migrated along the neurites and neurites that projected into the cancer cell colonies. (d-e-f) Upon contact, tumor cells disengage and navigate along the contacted neurites (blue arrowheads) toward DRG. Numbers indicate sites of neurite-tumor cell contact initiation and refer to corresponding magnified areas in (c). Original magnification, 100x; Scale bar $=100 \mu \mathrm{m}$. C. The travelling velocity of MGC803 control cells and MGC803 shNTN1 cells was calculated. D. The accumulated distance travelled by the MGC803 control cells and MGC803 shNTN1 cells was calculated. E. The average area covered by the neurites growing out from the DRG in different groups was quantified. ${ }^{*} \mathrm{p}<0.05,{ }^{* *} \mathrm{p}<0.01$, $* * * \mathrm{p}<0.001$.

human GC tumor tissues presence of NI exhibited a higher NTN1 expression level compared to normal GC tissues without NI. Because these varied roles may potentially intersect in NI, we hypothesized that NTN1 may play a role in this process.

Previous studies showed a prevalence of around $50-60 \%$ for NI in GC [11]. Understanding the relationship and underlying mechanisms of interactions between GC cells and related neural cells has become very important, because the clinical relevance of the tumor microenvironment to accelerate tumor progression has been widely recognized. To identify NTN1 and its receptors involved in NI, we performed a modified co-culture assay of DRG and GC cells. DRG explants in Matrigel sprout axonal-like tiny nerves, which in co-culture with cancer cells serve as a model that recapitulates NI [5, 10, 35-37]. Notably, our current data identified a novel, critical function of NTN1 for the interaction of GC cells with peripheral nerves. Through DRG-tumor cells co-culture assay, we indicated that NTN1 knockdown in GC cells inhibited their capacity to navigate along contacted neuritis in vitro. In addition, GC cells with NTN1 silencing were also seemed to fail 
to stimulate increased neurite density of DRG in contrast to the normal GC cells. Using mouse models, we also showed that NTN1 knockdown suppressed neural invasive potential of GC cells. Moreover, human GC specimens demonstrated a very high level of NTN1 expression (77.6\%) in tumors with NI. This correlation between the expression level of NTN1 and $\mathrm{NI}$ in human clinical specimens further supported the concept that NTN1 is likely playing a mechanistic role in the process of NI in GC.
NTN1 was a secreted protein that regulated axon guidance through its receptors [38]. In contrast to other NTN1 receptors, neogenin was found to be the most predominant in our GC cell lines. Recent studies have reported that neogenin was found in GC tissues and could promote GC cells migration and invasion abilities [39, 40]. It was also reported that NTN1/neogenin signaling may activate AKT/PI3K pathway to regulate cellular migration and invasion in GC cells [20]. Here, we observed that neogenin
A

\section{Adjacent tissue}

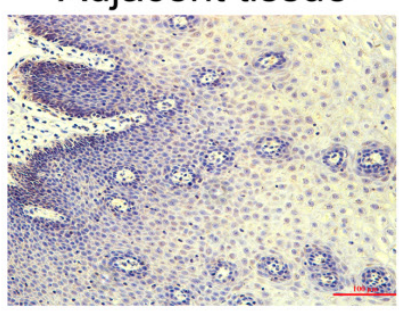

C

E

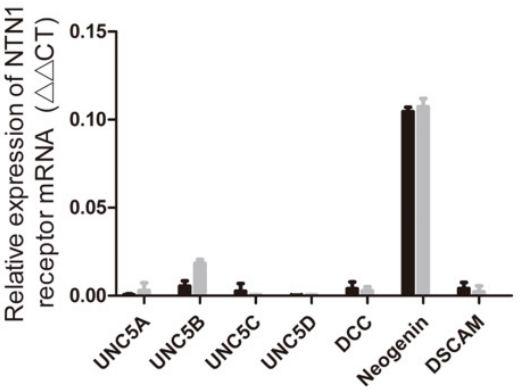

shCTL

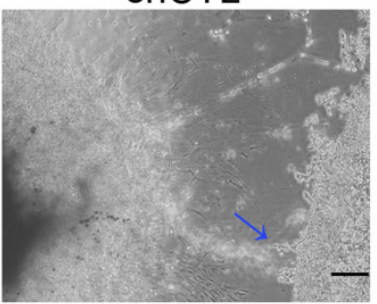

G

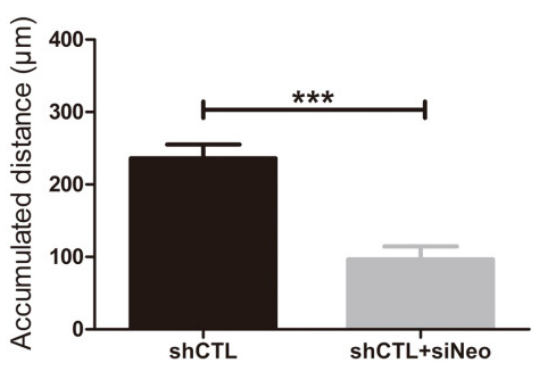

GC

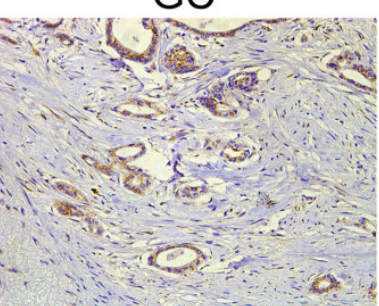

MGC803

MKN28
$\mathrm{B}$

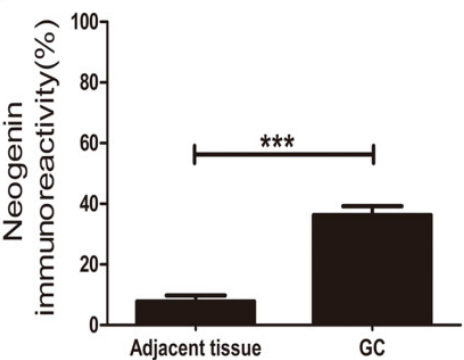

D

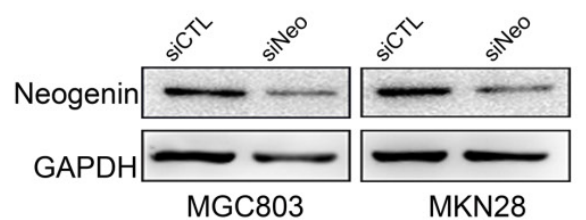

F

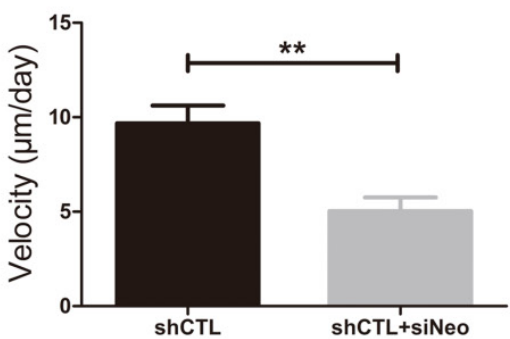

$\mathrm{H}$

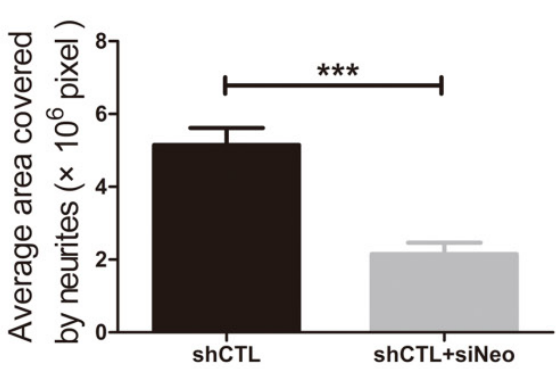

Fig. 5. NTN1 knockdown suppressed GC cells neural invasion was depend on neogenin. A. Representative images of neogenin IHC staining on sections from GC tissues and adjacent normal tissues. Original magnification, 200x; Scale bar=100 $\mu \mathrm{m}$. B. Quantification of the percentage of cells demonstrating the presence of immunoreactivity for neogenin (positive staining). C. The expression levels of NTN1 receptors in MGC803 and MKN28 cells were detected by qRT-PCR. D. Neogenin expression level was detected in GC cells by western blotting after transfecting with siNeogenin. E. Representative images of the migration of MGC803 cells towards nerves treated with/without neogenin inhibition. Blue arrowheads indicate the cancer cells that migrated towards the neurites. Original magnification, $40 \times ;$ Scale bar $=100 \mu m$. $\mathbf{F}$. The travelling velocity of MGC803 cells in different groups (shCTL and shCTL+siNeo) was calculated. $\mathbf{G}$. The accumulated distance travelled by the cancer cells in different groups was calculated. $\mathbf{H}$. The average area covered by the neurites growing out from the DRG in different groups was quantified. $*_{p}<0.05, * *_{p}<0.01, * * * p<0.001$. 
A

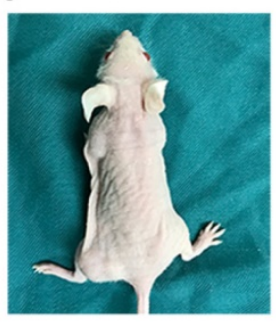

E

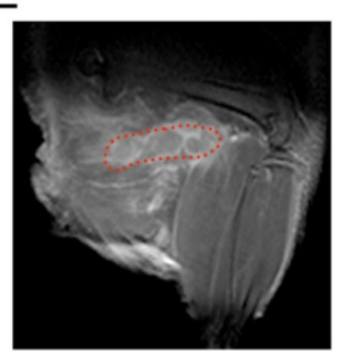

B

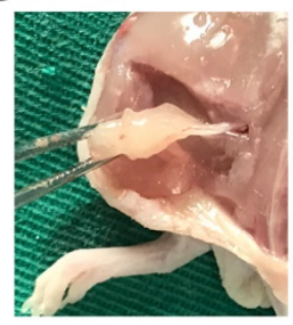

$\mathrm{F}$

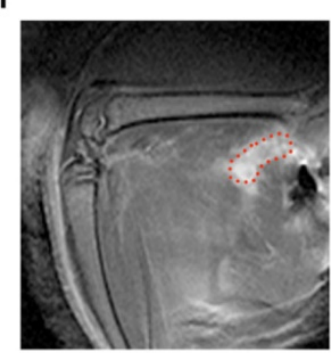

C

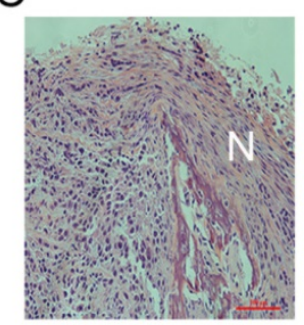

G

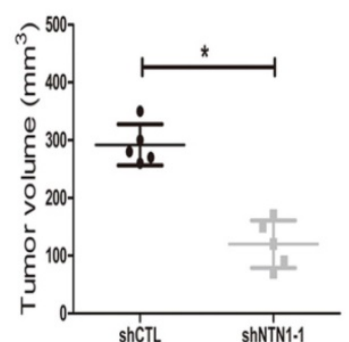

D

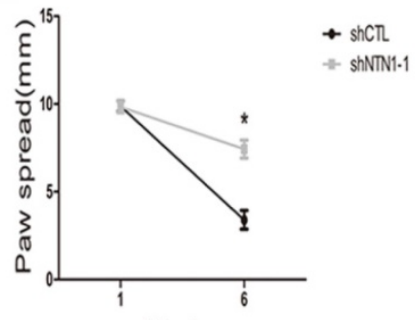

$\mathrm{H}^{\text {Week }}$

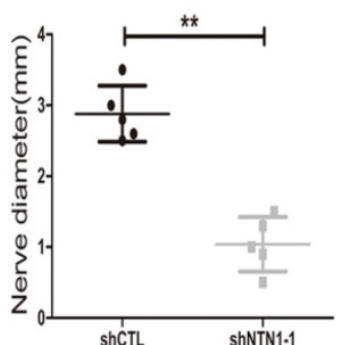

Fig. 6. Inhibition of NTN1 decreased GC cells neural invasion in vivo. A. In a sciatic nerve invasion model NTN1 inhibition prevented nerve destruction. Representative image of mice 6 weeks after tumor implantation showing left hind limb paralysis in the control group compared with normal function (right hind limb) in the NTN1 inhibition group. B-C. Representative images of extensive proximal nerve-invasion by GC cells. HE staining; N indicated nerve. D. NTN1 inhibition increased mice paw spread as compared with control group. E-F. Tumor volume and nerve diameter in different groups was measured by using a small animal Magnetic resonance. Representative images were showed (E-F). Red lines showed the extent of the tumor infiltrating the sciatic nerve. G-H. NTN1 inhibition decreased primary tumor volume in the sciatic nerve $(\mathrm{G})$ and decreased invasion through the nerve as determined by nerve diameter proximal to the primary tumor site $(\mathrm{H})$. ${ }^{*} \mathrm{p}<0.05$, $*^{*} \mathrm{p}<0.01$, ${ }^{* * *} \mathrm{p}<0.001$.

ablation could also diminish GC cells neural invasive abilities. In a word, NTN1 was required for GC cells related to neural outgrowth and enhancement of cancer cells NI and neogenin may be also involved in this process. Our findings showed the critical importance of the cancer studying neuroenvironment was a key regulatory factor of GC progression. Our study has some limitations. In this work, our results focused on one aspect of cancer-nerves relationship, and other factors including chemotatic factors, adhesion molecules and matrix metalloproteinase may be involved in this process.

In a word, we investigated the invasion of GC cells along DRG neuritis and demonstrated NTN1 and its receptor neogenin may play an important role in NI. Furthermore, we implied that this interaction likely result in motility for GC cells in the neural location. Considering the effect of NTN1 in neural invasion, future treatment direct against NI could theoretically prevent the local extension of GC to the peripheral nerve, reduce neuropathic pain and prolong patient survival.

\section{Acknowledgements}

This work was supported by the National Natural Science Foundation of China (NO. 81572362 and 81602080); Six talent peaks project in Jiangsu province(NO.2016-WSN-007); The Jiangsu province 333 project foundation(NO.BRA2017560); Zhenjiang
Key Research and Development Plan (NO. SH2017023, NO. SH2018035).

\section{Competing Interests}

The authors have declared that no competing interest exists.

\section{References}

1. Torre LA, Bray F, Siegel RL, Ferlay J, Lortet-Tieulent J, Jemal A. Global cancer statistics, 2012. CA Cancer J Clin. 2015; 65: 87-108.

2. Scanlon CS, Banerjee R, Inglehart RC, Liu M, Russo N, Hariharan A, et al. Galanin modulates the neural niche to favour perineural invasion in head and neck cancer. Nat Commun. 2015; 6: 6885 .

3. Ayala GE, Dai H, Powell M, Li R, Ding Y, Wheeler TM, et al. Cancer-Related Axonogenesis and Neurogenesis in Prostate Cancer. Clin Cancer Res. 2008; 14: 7593-603.

4. Ding Y, He D, Florentin D, Frolov A, Hilsenbeck S, Ittmann M, et al. Semaphorin 4F as a Critical Regulator of Neuroepithelial Interactions and a Biomarker of Aggressive Prostate Cancer. Clin Cancer Res. 2013; 19: 6101-11.

5. Liebl F, Demir IE, Rosenberg R, Boldis A, Yildiz E, Kujundzic K, et al. The severity of neural invasion is associated with shortened survival in colon cancer. Clin Cancer Res. 2013; 19: 50-61.

6. Abiatari I, DeOliveira T, Kerkadze V, Schwager C, Esposito I, Giese NA, et al. Consensus transcriptome signature of perineural invasion in pancreatic carcinoma. Mol Cancer Ther. 2009; 8: 1494-504.

7. Gil Z, Cavel O, Kelly K, Brader P, Rein A, Gao SP, et al. Paracrine regulation of pancreatic cancer cell invasion by peripheral nerves. J Natl Cancer Inst. 2010; 102: 107-18.

8. Dumartin L, Quemener C, Laklai H, Herbert J, Bicknell R, Bousquet C, et al. Netrin-1 mediates early events in pancreatic adenocarcinoma progression, acting on tumor and endothelial cells. Gastroenterology. 2010; 138: 1595-606, $606 \mathrm{e} 1-8$.

9. Demir IE, Ceyhan GO, Liebl F, D'Haese JG, Maak M, Friess H. Neural invasion in pancreatic cancer: the past, present and future. Cancers. 2010; 2: 1513-27.

10. Liebig C, Ayala G, Wilks JA, Berger DH, Albo D. Perineural invasion in cancer: a review of the literature. Cancer. 2009; 115: 3379-91.

11. Bapat AA, Hostetter G, Von Hoff DD, Han H. Perineural invasion and associated pain in pancreatic cancer. Nat Rev Cancer. 2011; 11: 695-707.

12. Lai Wing Sun K, Correia JP, Kennedy TE. Netrins: versatile extracellular cues with diverse functions. Development. 2011; 138: 2153-69.

13. Delloye-Bourgeois C, Fitamant J, Paradisi A, Cappellen D, Douc-Rasy S, Raquin MA, et al. Netrin-1 acts as a survival factor for aggressive neuroblastoma. J Exp Med. 2009; 206: 833-47. 
14. Huang Q, Hua HW, Jiang F, Liu DH, Ding G. Netrin-1 promoted pancreatic cancer cell proliferation by upregulation of Mdm2. Tumour Biol. 2014; 35: 9927-34.

15. Fitamant J, Guenebeaud C, Coissieux MM, Guix C, Treilleux I, Scoazec JY, et al. Netrin-1 expression confers a selective advantage for tumor cell survival in metastatic breast cancer. Proc Natl Acad Sci U S A. 2008; 105: 4850-5.

16. Kong CZ, Liu J, Liu L, Zhang Z, Guo KF. Interactional expression of netrin-1 and its dependence receptor UNC5B in prostate carcinoma. Tumour Biol. 2013; 34: 2765-72.

17. Delloye-Bourgeois C, Brambilla E, Coissieux MM, Guenebeaud C, Pedeux R, Firlej $\mathrm{V}$, et al. Interference with netrin-1 and tumor cell death in non-small cell lung cancer. J Natl Cancer Inst. 2009; 101: 237-47.

18. Han P, Fu Y, Liu J, Wang Y, He J, Gong J, et al. Netrin-1 promotes cell migration and invasion by down-regulation of BVES expression in human hepatocellular carcinoma. Am J Cancer Res. 2015; 5: 1396-409.

19. Ko SY, Blatch GL, Dass CR. Netrin-1 as a potential target for metastatic cancer: focus on colorectal cancer. Cancer Metastasis Rev. 2014; 33: 101-13.

20. Yin $\mathrm{K}$, Wang LJ, Zhang $\mathrm{X}, \mathrm{He} \mathrm{ZY}, \mathrm{Xia} \mathrm{YW}, \mathrm{Xu} \mathrm{JH}$, et al. Netrin-1 promotes gastric cancer cell proliferation and invasion via the receptor neogenin through PI3K/AKT signaling pathway. Oncotarget. 2017; 8: 51177-89.

21. Tian J, Rui K, Hong Y, Wang X, Xiao F, Lin X, et al. Increased GITRL Impairs the Function of Myeloid-Derived Suppressor Cells and Exacerbates Primary Sjogren Syndrome. J Immunol. 2019; 202: 1693-703.

22. Wang L, Zhi X, Zhu Y, Zhang Q, Wang W, Li Z, et al. MUC4-promoted neural invasion is mediated by the axon guidance factor Netrin-1 in PDAC. Oncotarget. 2015; 6: 33805-22.

23. Ceyhan GO, Demir IE, Altintas B, Rauch U, Thiel G, Muller MW, et al. Neural invasion in pancreatic cancer: a mutual tropism between neurons and cancer cells. Biochem Biophys Res Commun. 2008; 374: 442-7.

24. Naito S, von Eschenbach AC, Giavazzi R, Fidler IJ. Growth and metastasis of tumor cells isolated from a human renal cell carcinoma implanted into different organs of nude mice. Cancer Res. 1986; 46: 4109-15.

25. Kim SJ, Wang YG, Lee HW, Kang HG, La SH, Choi IJ, et al. Up-regulation of neogenin-1 increases cell proliferation and motility in gastric cancer. Oncotarget. 2014; 5: 3386-98.

26. Bilici A, Seker M, Ustaalioglu BB, Kefeli U, Yildirim E, Yavuzer D, et al. Prognostic significance of perineural invasion in patients with gastric cancer who underwent curative resection. Ann Surg Oncol. 2010; 17: 2037-44.

27. Arakawa H. Netrin-1 and its receptors in tumorigenesis. Nat Rev Cancer. 2004; 4: 978-87.

28. Akino T, Han X, Nakayama H, McNeish B, Zurakowski D, Mammoto A, et al. Netrin-1 promotes medulloblastoma cell invasiveness and angiogenesis, and demonstrates elevated expression in tumor tissue and urine of patients with pediatric medulloblastoma. Cancer Res. 2014; 74: 3716-26.

29. Chen $\mathrm{H}, \mathrm{Chen} \mathrm{Q}$, Luo Q. Expression of netrin-1 by hypoxia contributes to the invasion and migration of prostate carcinoma cells by regulating YAP activity. Exp Cell Res. 2016; 349: 302-9.

30. Grandin M, Meier M, Delcros JG, Nikodemus D, Reuten R, Patel TR, et al. Structural Decoding of the Netrin-1/UNC5 Interaction and its Therapeutical Implications in Cancers. Cancer cell. 2016; 29: 173-85.

31. Mazelin L, Bernet A, Bonod-Bidaud C, Pays L, Arnaud S, Gespach C, et al. Netrin-1 controls colorectal tumorigenesis by regulating apoptosis. Nature. 2004; 431: 80-4.

32. Paradisi A, Creveaux M, Gibert B, Devailly G, Redoulez E, Neves D, et al. Combining chemotherapeutic agents and netrin-1 interference potentiates cancer cell death. EMBO Mol Med. 2013; 5: 1821-34

33. Finci L, Zhang Y, Meijers R, Wang JH. Signaling mechanism of the netrin-1 receptor DCC in axon guidance. Prog Biophys Mol Biol. 2015; 118: 153-60.

34. Lv J, Sun X, Ma J, Ma X, Zhang Y, Li F, et al. Netrin-1 induces the migration of Schwann cells via p38 MAPK and PI3K-Akt signaling pathway mediated by the UNC5B receptor. Biochem Biophys Res Commun. 2015; 464: 263-8.

35. He $\mathrm{S}, \mathrm{He} \mathrm{S}$, Chen $\mathrm{CH}$, Deborde $\mathrm{S}$, Bakst RL, Chernichenko $\mathrm{N}$, et al. The chemokine (CCL2-CCR2) signaling axis mediates perineural invasion. Mol Cancer Res. 2015; 13: 380-90.

36. Gohrig A, Detjen KM, Hilfenhaus G, Korner JL, Welzel M, Arsenic R, et al. Axon Guidance Factor SLIT2 Inhibits Neural Invasion and Metastasis in Pancreatic Cancer. Cancer Res. 2014; 74: 1529-40.

37. Nomura A, Majumder K, Giri B, Dauer P, Dudeja V, Roy S, et al. Inhibition of NF-kappa B pathway leads to deregulation of epithelial-mesenchymal transition and neural invasion in pancreatic cancer. Lab Invest. 2016; 96: 1268-78.

38. Ren XR, Ming GL, Xie Y, Hong Y, Sun DM, Zhao ZQ, et al. Focal adhesion kinase in netrin-1 signaling. Nat Neurosci. 2004; 7: 1204-12.

39. Rodriguez A, Pan P, Parkkila S. Expression studies of neogenin and its ligand hemojuvelin in mouse tissues. J Histochem Cytochem. 2007; 55: 85-96.

40. Lv B, Song C, Wu L, Zhang Q, Hou D, Chen P, et al. Netrin-4 as a biomarker promotes cell proliferation and invasion in gastric cancer. Oncotarget. 2015; 6: 9794-806. 